\title{
Modelling in numerical simulation of electromagnetic heating
}

\author{
J. Rappaz and M. Swierkosz* \\ Department of Mathematics, Swiss Federal Institute of Technology \\ CH-1015 Lausanne, Switzerland. Phone: +412169325 55. \\ Fax: +412169343 03. E-mail: marek@dma.epfl.ch
}

\begin{abstract}
This paper deals with numerical simulation of induction heating for tri-dimensional timevarying axisymmetric geometries. The modelling used for the eddy current solver is presented in detail, from physical equations to a numerical scheme.
\end{abstract}

\section{Keywords}

Numerical simulation, electromagnetic heating, coupled problems, finite element method, boundary element method

\section{INTRODUCTION}

Induction heating involves both electromagnetic and thermal phenomena, described by coupled nonlinear partial differential equations. An induction heating setup usually consists of one or several inductors and one or several workpieces to be heated (Figure 1). The inductors may move with respect to the workpieces.

The alternating current flowing through the inductor generates a rapidly oscillating magnetic field. This in turn induces eddy currents inside the workpiece, which results in temperature increase due to the Joule effect and to the hysteresis effect.

In this research, we consider axisymmetric induction heating setups. The aim is to obtain a model which leads to an efficient numerical simulation of the induction heating phenomenon. It is assumed that the coils are supplied with a sinusoidal alternating current, so as to obtain a steady-state electromagnetic problem. The total voltage in each coil $v_{k}$ and the angular frequency $\omega$ are considered to be given.

\footnotetext{
*This research was supported by the Swiss "Nationaler Energie-Forschungs-Fonds" and performed in collaboration with the company Amysa Yverdon SA, Switzerland.
} 


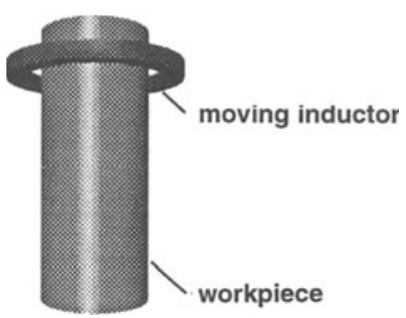

Figure 1 A sample induction heating setup

\section{MATHEMATICAL MODEL}

\subsection{Scalar magnetic potential formulation}

Let us consider an axisymmetric induction heating setup consisting of $N$ conductors (inductors and workpieces). Let $\left(\Delta_{i}\right)_{i=1}^{N}$ be $N$ bounded open sets of $\mathbf{R}^{3}$ corresponding to the areas in space occupied by the conductors. These sets are obtained by revolution of $N$ open simply connected sets $\Omega_{i} \subset \mathbf{R}^{2}, i=1, \ldots, N$, around a straight line that we consider to be the $O z$ axis of a Cartesian coordinate system $(x, y, z)$. Let us denote by $\Delta$ the union of the sets $\Delta_{i}: \Delta=\cup_{i=0}^{N} \Delta_{i}$, by $\Omega$ the union of the sets $\Omega_{i}$ : $\Omega=\cup_{i=0}^{N} \Omega_{i}$ and by $\Delta^{\prime}$ the complementary of $\bar{\Delta}$ in $\mathbf{R}^{3}(\bar{\Delta}$ denotes the adherence of $\Delta)$.

The starting point of our modelling are Maxwell equations with displacement currents neglected, and Ohm law. The following equations hold in whole $\mathbf{R}^{3}$ :

$$
\begin{aligned}
\operatorname{div} \mathbf{B} & =0 \\
\operatorname{curl} \mathbf{E} & =-\frac{\partial \mathbf{B}}{\partial t}, \\
\operatorname{curl} \mathbf{H} & =\mathbf{j} \\
\nu \mathbf{B} & =\mathbf{H}
\end{aligned}
$$

Moreover, in $\Delta$, we have:

$\mathbf{j}=\sigma \mathbf{E}$.

Here $t$ denotes the time, $\mathbf{E}$ the electric field, $\mathbf{H}$ the magnetic field, $\mathbf{B}$ the magnetic induction, $\mathbf{j}$ the electric current density, $\sigma$ the electric conductivity, and $\nu$ the inverse of the magnetic permeability $\mu$ (called magnetic reluctivity). For the moment, we assume that the values of $\sigma, \mu$ and $\nu$ do not depend on the time. In the reality, they will usually vary with the temperature and to a certain extent with the magnetic field. 
Let us now consider a cylindrical coordinate system $(r, \theta, z)$ with its associated natural tangent reference system $\left(\mathbf{e}_{r}, \mathbf{e}_{\theta}, \mathbf{e}_{z}\right)$, The following assumptions are made:

1. The fields $\mathbf{B}, \mathbf{H}, \mathbf{E}$ are invariant along $\theta$, i.e. their components in the reference system $\left(\mathbf{e}_{r}, \mathbf{e}_{\theta}, \mathbf{e}_{z}\right)$ do not depend on $\theta$.

2. The electric current density is of the form $\mathbf{j}=\mathbf{j}(\mathbf{r}, \mathbf{z}) \mathbf{e}^{\mathbf{i} \omega \mathbf{t}} \mathbf{e}_{\theta}$, where $j:(r, z) \in \mathbf{R}^{+} \times \mathbf{R} \mapsto$ $\mathbf{j}(\mathbf{r}, \mathbf{z}) \in \mathbf{C}$ is some complex-valued function.

It will also be assumed that there are no surface currents i.e. no Dirac $\delta$-like current "concentration" on the surface of the conductors.

Suppose that a periodic voltage of the form $v_{k} e^{i \omega t}$ is imposed in each of those sets $\Delta_{k}$ which are not simply connected. Those sets are necessarily toroidal and correspond to the inductor coils. In those sets $\Delta_{k}$ that are simply connected, we shall set $v_{k} \equiv 0$ by convention. Due to the linearity of the problem (with constant coefficients $\sigma, \mu$ and $\nu$ ), we can look for the fields $\mathbf{B}, \mathbf{H}, \mathbf{E}$ in the form:

$\mathbf{B}=\mathbf{B}(\mathbf{r}, \mathbf{z}) \mathrm{e}^{\mathrm{i} \omega \mathrm{t}}, \quad \mathbf{H}=\mathbf{H}(\mathbf{r}, \mathbf{z}) \mathrm{e}^{\mathrm{i} \omega \mathrm{t}}, \quad \mathbf{E}=\mathbf{E}(\mathbf{r}, \mathbf{z}) \mathrm{e}^{\mathrm{i} \omega \mathrm{t}}$

Here $\mathbf{B}:(\mathbf{r}, \mathbf{z}) \in \mathbf{R}^{+} \times \mathbf{R} \mapsto \mathbf{B}(\mathbf{r}, \mathbf{z}) \in \mathbf{C}^{\mathbf{3}}, \mathbf{H}:(\mathbf{r}, \mathbf{z}) \in \mathbf{R}^{+} \times \mathbf{R} \mapsto \mathbf{H}(\mathbf{r}, \mathbf{z}) \in \mathbf{C}^{\mathbf{3}}$, $\mathbf{E}:(\mathbf{r}, \mathbf{z}) \in \mathbf{R}^{+} \times \mathbf{R} \mapsto \mathbf{E}(\mathbf{r}, \mathbf{z}) \in \mathbf{C}^{\mathbf{3}}$ are complex vector fields to be found. In the sequel, we shall omit the term $e^{i \omega t}$.

Our aim is now to show that under the above assumptions, the magnetic induction $\mathbf{B}$ can be expressed in terms of a scalar potential $\phi:(r, z) \in \mathbf{R}^{+} \times \mathbf{R} \mapsto \phi(\mathbf{r}, \mathbf{z}) \in \mathbf{C}$.

Let us consider equation (3) expressed in cylindrical coordinates. According to Assumption 1 , the components of $\mathbf{H}$ in the natural tangent reference system are $\mathbf{H}=$ $\left(\mathbf{H}_{\mathbf{r}}(\mathbf{r}, \mathbf{z}), \mathbf{H}_{\theta}(\mathbf{r}, \mathbf{z}), \mathbf{H}_{\mathbf{z}}(\mathbf{r}, \mathbf{z})\right)$.

Therefore, equation (3) can be rewritten in the form

$$
\left(-\frac{\partial H_{\theta}}{\partial z}\right) \mathbf{e}_{r}-\left(\frac{\partial H_{z}}{\partial r}-\frac{\partial H_{r}}{\partial z}\right) \mathbf{e}_{\theta}+\left(\frac{1}{r} \frac{\partial\left(r H_{\theta}\right)}{\partial r}\right) \mathbf{e}_{z}=\mathbf{j} .
$$

Using assumption 2, we get the following system:

$$
\begin{aligned}
-\frac{\partial H_{\theta}}{\partial z} & =0 \\
-\frac{\partial H_{z}}{\partial r}+\frac{\partial H_{r}}{\partial z} & =j(r, z), \\
\frac{1}{r} \frac{\partial\left(r H_{\theta}\right)}{r} & =0 .
\end{aligned}
$$

Equation (8) implies that $H_{\theta}=H_{\theta}(r)$, and from (10) we get $H_{\theta}=\frac{c}{r}$, where $c$ is a constant. If $c$ were nonzero, we would have $\lim _{r \rightarrow 0} H_{\theta}=\infty$, which is absurd. Therefore, the magnetic field $\mathbf{H}$ has the form

$\mathbf{H}=\mathbf{H}_{\mathbf{r}}(\mathbf{r}, \mathbf{z}) \mathbf{e}_{\mathbf{r}}+\mathbf{H}_{\mathbf{z}}(\mathbf{r}, \mathbf{z}) \mathbf{e}_{\mathbf{z}}$ 
This result and equation (4) together imply that the magnetic induction $\mathbf{B}$ also has the form

$\mathbf{B}=\mathbf{B}_{\mathbf{r}}(\mathbf{r}, \mathbf{z}) \mathbf{e}_{\mathbf{r}}+\mathbf{B}_{\mathbf{z}}(\mathbf{r}, \mathbf{z}) \mathbf{e}_{\mathbf{z}}$

Equation (1) yields thus

$\frac{\partial}{\partial r}\left(r B_{r}\right)+\frac{\partial}{\partial z}\left(r B_{z}\right)=0$.

Equation (13) states the fact that the field $(r, z) \in \mathbf{R}^{+} \times \mathbf{R} \mapsto\left(\mathbf{r} \mathbf{B}_{\mathbf{r}}, \mathbf{r B}_{\mathbf{z}}\right) \in \mathbf{C}^{\mathbf{2}}$ is divergence-free when we consider $(r, z)$ as Cartesian coordinates. A well-known result (see e.g. Dautray and Lions, 1988) allows us to conclude that this field can be expressed in terms of the curl of a scalar function. In other words, there exists a function $\psi:(r, z) \in$ $\mathbf{R}^{+} \times \mathbf{R} \mapsto \psi(\mathbf{r}, \mathbf{z}) \in \mathbf{C}$ such that:

$r B_{r}=-\frac{\partial \psi}{\partial z}, \quad r B_{z}=\frac{\partial \psi}{\partial r}$.

Let $\phi:(r, z) \in \mathbf{R}^{+} \times \mathbf{R} \mapsto \phi(\mathbf{r}, \mathbf{z}) \in \mathbf{C}$ be defined by $\phi(r, z)=\frac{1}{r} \psi(r, z)$. Equations (14) can then be rewritten in the form:

$B_{r}=-\frac{\partial \phi}{\partial z}, \quad B_{z}=\frac{1}{r} \frac{\partial(r \phi)}{\partial r}$.

We conclude that there exists a vector magnetic potential $\mathbf{A}$ of the form

$\mathbf{A}(\mathbf{r}, \mathbf{z})=\phi(\mathbf{r}, \mathbf{z}) \mathbf{e}_{\theta}$

such that

$\mathbf{B}=\operatorname{curl} \mathbf{A}$.

Moreover, we clearly have

$\operatorname{div} \mathbf{A}=\mathbf{0}$,

since $\operatorname{div} \mathbf{A}=\frac{\mathbf{1}}{\mathbf{r}} \frac{\partial \phi}{\partial \theta}$. Using Biot-Savart law, one can show that $\mathbf{B} \sim \frac{\mathbf{1}}{\left(\mathbf{r}^{\mathbf{2}}+\mathbf{z}^{2}\right)^{\frac{3}{2}}}$ when $\left(r^{2}+z^{2}\right)^{\frac{1}{2}} \rightarrow \infty$. In (14), $\psi$ is defined up to a constant. Consequently, $\phi$ can be chosen so that $\phi \sim \frac{1}{r^{2}+z^{2}}$ when $\left(r^{2}+z^{2}\right)^{\frac{1}{2}}$ tends to infinity, or, in other words,

$\phi=O\left(\frac{1}{r^{2}+z^{2}}\right)$ when $|\mathrm{r}|+|z| \rightarrow \infty$.

Taking into account (17), (4) and Assumption 2, equation (3) yields

$\operatorname{curl}(\nu \operatorname{curl} \mathbf{A})=\mathbf{j e}_{\theta}$, 
which can be expanded to the form

$-\left(\frac{\partial}{\partial r}\left(\frac{\nu}{r} \frac{\partial(r \phi)}{\partial r}\right)+\frac{\partial}{\partial z}\left(\nu \frac{\partial \phi}{\partial z}\right)\right) \mathbf{e}_{\theta}=\mathbf{j}$.

This result holds both inside the conductors and outside them.

Outside the conductors, $j$ is zero and $\nu$ is constant, so that (21) yields

$\frac{\partial}{\partial r}\left(\frac{1}{r} \frac{\partial(r \phi)}{\partial r}\right)+\frac{\partial^{2} \phi}{\partial z^{2}}=0$

This equation does not imply that $\Delta \phi=0$. However, multiplying it by $\sin \theta$, we get

$\Delta(\phi \sin \theta)=0$.

\subsection{Eddy current model}

So far, we have expressed the magnetic field and the magnetic induction in terms of a scalar magnetic potential $\phi$. Our aim is now to find a relationship between the current density $j$, the potential $\phi$, and the voltage $v_{k}$ imposed in the conductor.

From Ohm law (5) and Assumption 2, we get that $\mathbf{E}=\mathbf{E}(\mathbf{r}, \mathbf{z}) \mathbf{e}_{\theta}$ in each open set $\Delta_{k}$ (corresponding to the location of a conductor). By rewriting the equation (2) in cylindrical coordinates, taking into account (6) and (12), we get that

$i \omega\left(B_{r} \mathbf{e}_{r}+B_{z} \mathbf{e}_{z}\right)+\left(-\frac{\partial E}{\partial z}\right) \mathbf{e}_{r}+\left(\frac{1}{r} \frac{\partial(r E)}{\partial r}\right) \mathbf{e}_{z}=0$

i.e. $i \omega B_{r}=\frac{\partial E}{\partial z}, i \omega B_{z}=-\frac{1}{r} \frac{\partial(r E)}{\partial r}$. Substituting (15) into these equations, we get

$\frac{\partial}{\partial z}(r E+i \omega r \phi)=0, \quad \frac{\partial}{\partial r}(r E+i \omega r \phi)=0$.

Consequently, there exist constants $c_{k} \in \mathbf{C}, \mathbf{k}=\mathbf{0}, . ., \mathbf{N}$ such that

$r E+i \omega r \phi=c_{k}$ in $\Delta_{\mathrm{k}}, \quad \mathrm{k}=0, . ., \mathrm{N}$.

Ohm law (5) yields

$j=\sigma\left(-i \omega \phi+\frac{c_{k}}{r}\right), \quad k=1, . ., N$.

Let $\Delta_{k}$ be a toroidal conductor to which a voltage $v_{k}$ is applied. Then, there exists a disk $\Sigma_{k}$, centered on the $O z$ axis of the cylindrical coordinates system and orthogonal to this axis, such that the $\Delta_{k} \cup \Sigma_{k}$ is simply connected (Figure 2). The voltage $v_{k}$ is then defined as a line integral of $\mathbf{E}+\mathrm{i} \omega \mathbf{A}$ along $\partial \Sigma_{k}$. Since $\mathbf{A}$ takes the form (16), we get: $v_{k}=\int_{\partial \Sigma_{k}}(E+i \omega \phi) \mathbf{e}_{\theta} \mathbf{d} \tau$. Taking into account (26) and the fact that $\mathbf{d} \tau=\mathbf{e}_{\theta} \mathbf{r d} \theta$ in this case, we obtain: $v_{k}=\int_{0}^{2 \pi} c_{k} d \theta=2 \pi c_{k}$. Therefore, in any non simply connected set $\Delta_{k}$, we have

$j=\sigma\left(-i \omega \phi+\frac{v_{k}}{2 \pi r}\right)$. 


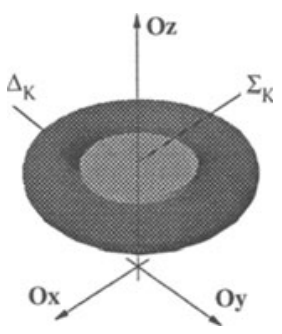

Figure 2 Application of a voltage to a toroidal conductor

Any simply connected axisymmetric set must have a non-empty intersection with the $O z$ axis, where $r$ is zero. Since the current density cannot be infinite, equation (27) implies that $c_{k}$ is zero in those of the sets $\Delta_{k}$ that are simply connected.

Let us remark that an inductor cannot be simply connected. In all workpieces, the voltage $v_{k}$ is zero, which implies that $c_{k}$ is zero also in those workpieces that are not simply connected.

Equations (28), (21) and Assumption 2 yield the following condition, valid in any conductor $\Delta_{k}$ :

$-\left(\frac{\partial}{\partial r}\left(\frac{\nu}{r} \frac{\partial(r \phi)}{\partial r}\right)+\frac{\partial}{\partial z}\left(\frac{\nu}{r} \frac{\partial(r \phi)}{\partial z}\right)\right)+i \omega \sigma \phi=\frac{\sigma v_{k}}{2 \pi r}$.

\subsection{Interface conditions}

We shall now attempt to find conditions holding on the boundary of the conductors. Let $[f]$ denote the jump of a function $f$ on $\partial \Delta$, and let $\mathbf{n}=\left(\mathbf{n}_{\mathbf{r}}, \mathbf{n}_{\mathbf{z}}\right)$ be the normal vector to $\partial \Delta$. Equations (16) and (17) imply that $\phi$ must be continuous. Since we assumed that there are no surface currents, we have $[\mathbf{H} \times \mathbf{n}]=\mathbf{0}$ on $\partial \Delta$. Since $\mathbf{H} \times \mathbf{n}=\left(\mathbf{H}_{\mathbf{z}} \mathbf{n}_{\mathbf{r}}-\mathbf{H}_{\mathbf{r}} \mathbf{n}_{\mathbf{z}}\right) \mathbf{e}_{\theta}$, we get $\left[H_{r} n_{z}-H_{z} n_{r}\right]=0$. Equations (15) and (4) yield $\left[\frac{\nu}{r}\left(\frac{\partial(r \phi)}{\partial z} n_{z}+\frac{\partial(r \phi)}{\partial r} n_{r}\right)\right]=0$, or shortly $\left[\frac{\nu}{r} \frac{\partial(r \phi)}{\partial n}\right]=0$. We have thus the following interface conditions:

$[\phi]=\left[\frac{\nu}{r} \frac{\partial(r \phi)}{\partial n}\right]=0$

In conclusion, the model consists of equation (22) (or 23) in $\Delta^{\prime}$ (outside the conductors), equation (29) in $\Delta$ (inside the conductors), interface condition (30) and condition (19) at infinity. 


\section{NUMERICAL MODEL}

Our aim is now to formulate a set of equations that could be used in a numerical simulation code. The difficulty lies in the fact that equation (22) is defined over an unbounded domain. Therefore a straightforward solution by finite element method is not possible.

Equation (22) could be solved over the whole unbounded domain using so-called "infinite elements". Another commonly found way of dealing with such problems is to approach the infinite domain by a "sufficiently large" finite domain, with suitable conditions at the boundary, and to solve the whole problem by standard finite element method.

In our case, these two solutions must be ruled out. In fact, the assumption is that inductors may move with respect to the workpieces. Therefore, the mesh outside the conductors would have to vary with the inductor movement. Apart from other considerations, the generation of such a mesh would lead to efficiency problems.

We opted therefore for a mixed boundary element - finite element formulation. The behaviour of the magnetic potential outside the conductors will be expressed in terms of integrals over their boundary, while inside the conductors a classical finite element formulation was opted for.

The key of the boundary element part is the so-called simple-double layer formulation. Let $G(y, x)=\frac{1}{4 \pi|x-y|}$ denote the Green kernel in $\mathbf{R}^{\mathbf{3}}$. Let $f$ denote any function of $C^{2}\left(\mathbf{R}^{\mathbf{3}}\right)$, harmonic in $\Delta^{\prime}$ and such that $f=O\left(\frac{1}{|x|}\right)$ when $|x| \rightarrow \infty$. Then, the value of $f$ at any point $y \in \partial \Delta$ is given by the equation (Nédélec, 1977):

$$
\frac{f(y)}{2}=\int_{\partial \Delta} \frac{\partial f(x)}{\partial n_{x}} G(y, x) d s_{x}-\int_{\partial \Delta} \frac{\partial G(y, x)}{\partial n_{x}} f(x) d s_{x}
$$

where $\mathbf{n}_{\mathbf{x}}$ denotes the normal vector to $\partial \Delta$ at the point $x$, oriented outside $\Delta^{\prime}$.

We apply the above formulation to the function $\phi \sin \theta$ which satisfies equation (23). Let the points $x, y \in \mathbf{R}^{3}$ be denoted by $x=(r, \theta, z), y=\left(r_{b}, \theta_{b}, z_{b}\right)$ in cylindrical coordinates. Then the Green kernel is

$$
G\left(r_{b}, \theta_{b}, z_{b}, r, \theta, z\right)=\frac{1}{4 \pi} \frac{1}{\left[\left(r_{b} \cos \theta_{b}-r \cos \theta\right)^{2}+\left(r_{b} \sin \theta_{b}-r \sin \theta\right)^{2}+\left(z_{b}-z\right)^{2}\right]^{1 / 2}} .
$$

Since the normal vector to $\partial \Delta$ has no component along $\theta$, we have $\frac{\partial(\sin \theta)}{\partial n_{x}}=0$. Therefore, equation (31) takes the form

$$
\frac{\phi\left(r_{b}, z_{b}\right) \sin \theta_{b}}{2}=\int_{\partial \Delta} \frac{\partial(\phi(r, z) \sin \theta)}{\partial n_{x}} G(y, x) d s_{x}-\int_{\partial \Delta} \frac{\partial G(y, x)}{\partial n_{x}} \phi(r, z) \sin \theta d s_{x} .
$$

Since the problem is axisymmetric, we can choose an arbitrary value for $\theta_{b}$ in the above equation. As $\Delta=\Omega \times[0,2 \pi]$, we get:

$$
\begin{aligned}
\phi\left(r_{b}, z_{b}\right) & =\frac{1}{2 \pi} \int_{\partial \Omega} \frac{\partial \phi(r, z)}{\partial n_{x}} g\left(r, z, r_{b}, z_{b}\right)-\phi(r, z) \tilde{g}\left(r, z, r_{b}, z_{b}\right) d s_{r, z}, \text { where } \\
g\left(r, z, r_{b}, z_{b}\right) & =4 \pi \int_{0}^{2 \pi} G\left(r, \theta, z, r_{b}, \frac{\pi}{2}, z_{b}\right) \sin \theta d \theta
\end{aligned}
$$


$\tilde{g}\left(r, z, r_{b}, z_{b}\right)=4 \pi \int_{0}^{2 \pi} \frac{\left.\partial G\left(r, \theta, z, r_{b}, \frac{\pi}{2}, z_{b}\right)\right)}{\partial n_{x}} \sin \theta d \theta$.

The integrands in (35) and (36) have an elementary expression in terms of $r, r_{b}, z, z_{b}, \theta$ and n. However, computation of integrals involving these functions gives rise to challenging numerical problems which are beyond the scope of this paper.

Equations (34) and (29) give rise to a finite element approximation. A triangular mesh $\tau_{h}$ is built over $\Omega$. A mesh on the boundary $\partial \Omega$ is induced by $\tau_{h}$. Since the normal derivative of $\phi$ is not continuous on $\partial \Omega$, we introduce a new variable $\lambda=\frac{\nu}{r} \frac{\partial(r \phi)}{\partial n}$ which is continuous on $\partial \Omega$ according to (30). To approximate $\phi$, we use standard $\mathbf{P}_{1}$ elements inside $\Omega$, while $\mathbf{P}_{0}$ elements are used to approximate $\lambda$ on $\partial \Omega$. We obtain thus a linear system where the unknowns are the values of $\phi$ at the mesh nodes and the values of $\lambda$ on the boundary edges. We obtain thus a boundary element-finite element model which was implemented into an eddy current computation code.

\section{SIMULATION SOFTWARE}

The eddy current computation code presented above was coupled with a module computing the temperature evolution. The heat equation, together with suitable radiation and convection conditions on the boundary is solved by standard finite elements, using an enthalpic formulation and taking into account the possible phase transformations. An additional module computing solid state phase transformations in steels was added to the whole software. This allows to simulate stream quenching of steels.

The simulation algorithm can be summarized as follows: we solve first the electromagnetic problem for a given time $t_{0}$ and a given initial temperature field. We suppose that this solution is valid for a short time step $\tau$, during which the temperature variation did not influence too much the physical characteristics of the conductors. We solve then the thermal problem over the interval $\left[t_{0}, t_{0}+\tau\right]$. We update the physical characteristics of the conductors according to the new temperature field, and we also update the coordinates of the inductor in case of inductor movement. We proceed then to another solution of the electromagnetic problem. The whole procedure is repeated as many times as necessary.

An' account of this work, as well as comparisons of numerical results with experiments, can be found in Jacot, Swierkosz et al.(1995). The software is now being used in industrial practice.

\section{REFERENCES}

Jacot, A., Swierkosz, M., Rappaz, J., Rappaz, M. and Mari, D. (1995) Modelling of Electromagnetic Heating, Cooling and Phase Transformations During Surface Hardening of Steels, submitted to: Proceedings of Mecamat'95, La Bresse, France, May 16-19.

R. Dautray, J.-L. Lions, Analyse mathématique et calcul numérique pour les sciences et les techniques, Masson, Paris.

Nédélec, J.C. (1977) Approximation des équations intégrales en mécanique et en physique, cours du Centre de Mathématiques Appliquées, Ecole Polytechnique, Paris. 